Check for updates

Cite this: RSC Adv., 2017, 7, 50246

Received 21st August 2017

Accepted 23rd October 2017

DOI: $10.1039 / \mathrm{c} 7 \mathrm{ra09231h}$

rsc.li/rsc-advances

\section{Cobalt sulfide supported on nitrogen and sulfur dual-doped reduced graphene oxide for highly active oxygen reduction reaction $\uparrow$}

\author{
Ying Zhang, $\ddagger^{\mathrm{a}}$ Pingwei Li, $\ddagger^{\mathrm{a}}$ Xuying Yin, ${ }^{\mathrm{a}}$ Ya Yan, ${ }^{\mathrm{ab}}$ Ke Zhan, ${ }^{\mathrm{a}} \mathrm{Junhe}$ Yang ${ }^{\mathrm{ab}}$ \\ and Bin Zhao (D) *ab
}

Cobalt sulfide nanoparticles grown on nitrogen and sulfur dual-doped reduced graphene oxide sheets (COS/NS-rGO) were synthesized as an efficient electrocatalyst for the oxygen reduction reaction (ORR) by a facile one-step annealing process at $400-600{ }^{\circ} \mathrm{C}$. The catalyst synthesized at $500{ }^{\circ} \mathrm{C}(\mathrm{Co}-\mathrm{S} / \mathrm{NS}-\mathrm{rGO}-$ 500) exhibits the best ORR catalytic activity compared to the other samples, together with high fourelectron selectivity and excellent stability in alkaline medium. Moreover, the Co-S/NS-rGO-500 composite also manifests good ORR activity and selectivity in acid solution. The facile synthesis approach and superior ORR performance in both alkaline and acid electrolytes make the Co-S/NS-rGO catalysts promising as an alternative to commercial Pt/C catalyst for fuel cells.

\section{Introduction}

On account of the impending global energy crisis, fuel cells have become attractive as a promising power source with high energy conversion efficiency, high power density, quiet operation and low emission. ${ }^{\mathbf{1} 2}$ However, the high cost of common Pt-based electrocatalysts and sluggish kinetics of the oxygen reduction reaction (ORR) at the cathode are still the major limiting factors for commercialization of fuel cells. ${ }^{3,4}$ Therefore, development of inexpensive non-precious metal electrocatalysts that rival the ORR performance of Pt catalysts remains a big challenge.

Over the past decades, tremendous efforts have been devoted to replacing Pt-based catalysts by employing heteroatom-doped carbon nanomaterials as potential candidates for ORR catalysts. $^{5-9}$ Although nitrogen-doped (N-doped) graphene appears to be promising as a potential ORR electrocatalyst due to high catalytic activity and low cost, ${ }^{\mathbf{1 0 , 1 1}}$ most mono-heteroatom doped carbon materials still show inferior activity to that of the $\mathrm{Pt} / \mathrm{C}$ catalyst. More recently, dual-doped graphene with more than one heteroatom, such as P, N dual-doped, and S, N dual-doped graphene, has been proved to be more active for ORR than $\mathrm{N}$ doped graphene because of the advantages of different heteroatoms in conjugated carbon backbone which can generate more active sites and the synergistic effect between the dopants. ${ }^{12,13}$ Besides, cobalt sulfides with different stoichiometric ratios have

${ }^{a}$ School of Materials Science and Engineering, University of Shanghai for Science and Technology, Shanghai 200093, China.E-mail: zhaobin@usst.edu.cn

${ }^{b}$ Shanghai Innovation Institute for Materials, Shanghai 200444, China

$\dagger$ Electronic supplementary information (ESI) available. See DOI: 10.1039/c7ra09231h

\$ These authors contributed equally to this work. been previously explored as ORR catalysts and exhibit attractive electrocatalytic performance among all non-precious metal chalcogenides. ${ }^{14-17}$ However, the low electrical conductivity and the easy agglomeration of cobalt sulfides often result in the unsatisfactory ORR activities. ${ }^{\mathbf{1 4 , 1 5 , 1 8}}$ Therefore, nanocomposite with cobalt sulfides grown on conductive graphene support appears to a promising strategy for active ORR catalyst. Recently, Dai's group synthesized $\mathrm{Co}_{1-x} \mathrm{~S}$ nanoparticles on reduced graphene oxide (rGO) and found that the rGO supports facilitated growth of smaller $\mathrm{Co}_{1-x} \mathrm{~S}$ nanoparticles, leading to enhanced surface area and active catalytic sites for ORR. ${ }^{19}$ Higgins et al. reported improved ORR performance of $\mathrm{CoS}_{2}$ octahedral nanoparticles on $\mathrm{N}$ and $\mathrm{S}$ co-doped graphene/carbon nanotubes $\left(\mathrm{CoS}_{2}-\mathrm{CG}\right)$ than unsupported $\mathrm{CoS}_{2}$ catalyst, and demonstrated the importance of carbon supports in formation of the single crystal $\operatorname{CoS}_{2}$ nanoparticles. ${ }^{20}$ Despite of these advances in cobalt sulfide based non-precious metal ORR catalysts, the catalytic activities and long-term stability of most materials are still lower than those of the commercial Pt/ C. ${ }^{\mathbf{1 5 , 1 8 - 2 0}}$ Furthermore, complicated preparation procedure also increase cost of the nanocomposite ORR catalysts. Therefore, it is highly desirable to develop cobalt sulfide/graphene composite catalysts with high activity using simple synthesis method.

Herein, we propose a facile one-step annealing process to synthesize cobalt sulfide nanoparticles supported on N, S dualdoped graphene (Co-S/NS-rGO) as an efficient ORR catalyst. Due to the homogeneous distribution of cobalt sulfide nanoparticles, $\mathrm{N}$ and $\mathrm{S}$ dual-doping, as well as their synergistic effects, the nanocomposite catalyst exhibits excellent ORR catalytic activity and high four-electron selectivity in alkaline and acid media. 


\section{Experimental}

\subsection{Preparation of Co-S/NS-rGO}

Graphene oxide (GO) was used as a starting materials and made by a modified Hummers' method as described in literatures. ${ }^{\mathbf{1 9 , 2 0}}$ In a typical synthesis, $20 \mathrm{mg}$ ammonium thiocyanate $\left(\mathrm{NH}_{4} \mathrm{SCN}\right)$ and $5 \mathrm{mg}$ cobalt acetate $\left(\mathrm{Co}(\mathrm{OAC})_{2}\right)$ were firstly dissolved in $30 \mathrm{ml}$ deionized water. Secondly, $20 \mathrm{mg}$ GO was added into the precursor solution and ultrasonically dispersed for $2 \mathrm{~h}$. Subsequently, the mixture was further stirred for additional $48 \mathrm{~h}$ to achieve a homogeneous dispersion solution. Then, the solution was freezing dried to obtain intermediate product. Finally, the intermediate product was annealed at a temperature ranging between $400-600{ }^{\circ} \mathrm{C}$ for $2 \mathrm{~h}$ in Ar atmosphere, and the obtained material is designated as Co-S/NS-rGO-T (where $T$ stands for the annealing temperature). For comparison, nitrogen and sulfur dual-doped graphene (NS-rGO) was also prepared using the same method except the addition of cobalt acetate.

\subsection{Materials characterization}

Morphology of the Co-S/NS-rGO was observed by field-emission scanning electron microscope (FE-SEM, FEI, Quanta FEG 450) operated at 20-30 kV. Microstructure of the samples was examined by high resolution transmission electron microscopy (HRTEM; JEOL JEM-2010). X-ray diffraction (XRD) was employed to analyze crystal structure of the samples and performed on a Bruker D8-Advance X-ray diffractometer using a Cu $\mathrm{K} \alpha$ radiation $(\lambda=0.154 \mathrm{~nm})$. X-ray photoelectron spectroscopy (XPS, ESCALAB250Xi, Thermo Fisher) was carried out to evaluate chemical state and composition of the samples by using a $\mathrm{Mg} \mathrm{K} \alpha$ source. Raman spectra was recorded with a $785 \mathrm{~nm}$ laser as excitation source using a Raman Station (PerkinElmer 400).

\subsection{Electrochemical measurements}

Electrochemical performance of the Co-S/NS-rGO catalyst was measured at room temperature on an electrochemical workstation (CHI 760E, CH Instrument) in a conventional threeelectrode cell. A platinum wire, a saturated-calomel electrode (SCE), and catalyst-coated glassy carbon (GC) electrode were used as counter electrode, reference electrode, and working electrode, respectively, in $0.1 \mathrm{M} \mathrm{KOH}$ or $0.5 \mathrm{M} \mathrm{H}_{2} \mathrm{SO}_{4}$ solution. To prepare the catalyst ink, $1.5 \mathrm{mg}$ catalyst powder, $10 \mu \mathrm{l}$ Nafion (Alfa, $5 \mathrm{wt} \%$ ) and $190 \mu \mathrm{l}$ ethanol were mixed by ultrasonication for at least $30 \mathrm{~min}$. Subsequently, $10 \mu \mathrm{l}$ catalyst ink solution was pipetted on the GC electrode surface and dried at room temperature. The typical catalyst loading on the GC electrode is about $0.38 \mathrm{mg} \mathrm{cm}^{-2}$.

Cyclic voltammograms (CV), rotating disk electrode (RDE) and rotating ring-disk electrode (RRDE) techniques were utilized to characterize electrochemical activity of the catalysts. CVs were recorded in $\mathrm{O}_{2}$-saturated $\mathrm{KOH}\left(\mathrm{or} \mathrm{H}_{2} \mathrm{SO}_{4}\right.$ ) electrolyte solution within the potential range from $0.2 \mathrm{~V}$ to $-0.8 \mathrm{~V}$ (or from $-0.2 \mathrm{~V}$ to $0.8 \mathrm{~V}$ ), and the current-potential polarization curves of RDE studies were obtained at an electrode rotating speed of $900 \mathrm{rpm}$. Both CV and RDE experiments were performed at the potential scanning rate of $5 \mathrm{mV} \mathrm{s}^{-1}$. RRDE voltammograms were measured by setting the ring potential at $0.5 \mathrm{~V}$ in $0.1 \mathrm{M}$ $\mathrm{KOH}$ or $1.0 \mathrm{~V}$ in $0.5 \mathrm{M} \mathrm{H}_{2} \mathrm{SO}_{4}$ solution, while scanning the disk potential at a rate of $5 \mathrm{mV} \mathrm{s}^{-1}$. All potentials reported in this work are calibrated to the reversible hydrogen electrode (RHE) according to the literatures., ${ }^{2,21}$

\section{Results and discussion}

The facile preparation process and the structure of the Co-S/NSrGO composites are illustrated in Scheme 1. Briefly, $\mathrm{NH}_{4} \mathrm{SCN}$ and $\mathrm{Co}(\mathrm{OAC})_{2}$ are used as the nitrogen, sulfur and cobalt sources, respectively, which are mixed with GO in aqueous solution. Then, the dispersion solution is lyophilized to obtain the intermediate product. Subsequently, the dried mixture is annealed in $\mathrm{Ar}$ atmosphere to obtain the Co-S/NS-rGO composites. Since annealing temperature is known to be a key factor to influence the performance of the catalysts for ORR, ${ }^{21,22}$ the Co-S/NS-rGO composite catalyst are synthesized at various annealing temperatures to achieve the optimum electrocatalytic properties.

Crystal structure of the Co-S/NS-rGO composites synthesized at various temperatures was investigated by XRD. Interestingly, the annealing temperature was found to have significant influence on structure of the cobalt sulfide. As shown in Fig. 1, the broad peaks at about $25^{\circ}$ and $43^{\circ}$ correspond to the (002) and (100) diffraction of graphitic carbon, respectively, confirming the presence of rGO. $^{23}$ For the sample annealed at $400{ }^{\circ} \mathrm{C}$, the diffraction peaks at $32.3^{\circ}, 36.4^{\circ}, 39.9^{\circ}, 46.6^{\circ}, 55^{\circ}$, $60.2^{\circ}, 62.7^{\circ}$, and $70.5^{\circ}$ can be well assigned to cubic $\operatorname{CoS}_{2}$ phase (JCPDS no. 41-1471). ${ }^{21}$ When the annealing temperature is above $500{ }^{\circ} \mathrm{C}$ (both 500 and $600{ }^{\circ} \mathrm{C}$ ), the $\mathrm{CoS}_{2}$ peaks disappear and the new diffraction peaks appeared at $30.8^{\circ}, 35.4^{\circ}, 46.9^{\circ}$ and $54^{\circ}$ are attributed to $\mathrm{Co}_{1-x} \mathrm{~S}$ phase (JCPDS no. 42-0826) with hexagonal structure in space group $P 6_{3} / m m c$ (no. 194). ${ }^{\mathbf{1 9}}$ Therefore, a phase transition from cubic to hexagonal takes place for the cobalt sulfide as the annealing temperature rises from $400{ }^{\circ} \mathrm{C}$ to over $500{ }^{\circ} \mathrm{C}$.

Since both $\mathrm{CoS}_{2}$ and $\mathrm{Co}_{1-x} \mathrm{~S}$ have been reported to be active for ORR, ${ }^{2,20}$ the Co-S/NS-rGO composites fabricated here are expected to be good ORR electrocatalysts.

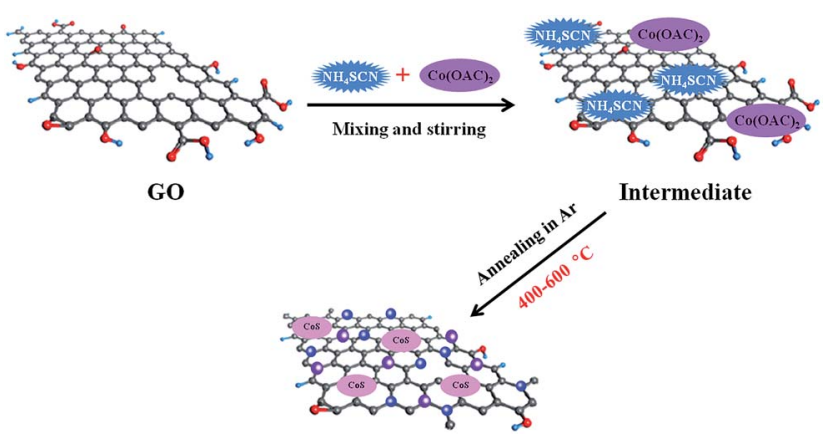

Scheme 1 Schematic illustration of the synthesis of Co-S/NS-rGO composite by one-step annealing. 




Fig. 1 XRD patterns of the catalysts: (a) Co-S/NS-rGO-400, (b) Co-S/ NS-rGO-500, (c) Co-S/NS-rGO-600.

Structural information about the Co-S/NS-rGO was also obtained from Raman analysis. Fig. 2 shows Raman spectra of pristine GO and the Co-S/NS-rGO composites synthesized at various temperatures, which presents two characteristic Raman peaks. The $\mathrm{G}$ band at $1590 \mathrm{~nm}^{-1}$ originates from the $\mathrm{sp}^{2}$ hybridized graphitic carbon, whereas the $\mathrm{D}$ band at $1350 \mathrm{~nm}^{-1}$ is associated with disordered carbon in GO. The intensity ratio of $\mathrm{D}$ band $\left(I_{\mathrm{D}}\right)$ and $\mathrm{G}$ band $\left(I_{\mathrm{G}}\right)$ is often used as an index of defect levels in carbon materials. ${ }^{24,25}$ Pristine GO has an $I_{\mathrm{D}} / I_{\mathrm{G}}$ ratio of 1.03, whereas the Co-S/NS-rGO composites demonstrate higher $I_{\mathrm{D}} / I_{\mathrm{G}}$ ratios (around 1.15), which can be attributed to the imperfections induced by the heteroatom doping in the graphene structure..$^{\mathbf{1 3 2 5 , 2 6}}$ Interestingly, the $I_{\mathrm{D}} / I_{\mathrm{G}}$ ratios decrease slightly with the increase in annealing temperature, indicating that the graphene structure is partially recovered at higher temperature.

Morphology and microstructure of the Co-S/NS-rGO composites were further examined by TEM. Fig. 3 shows

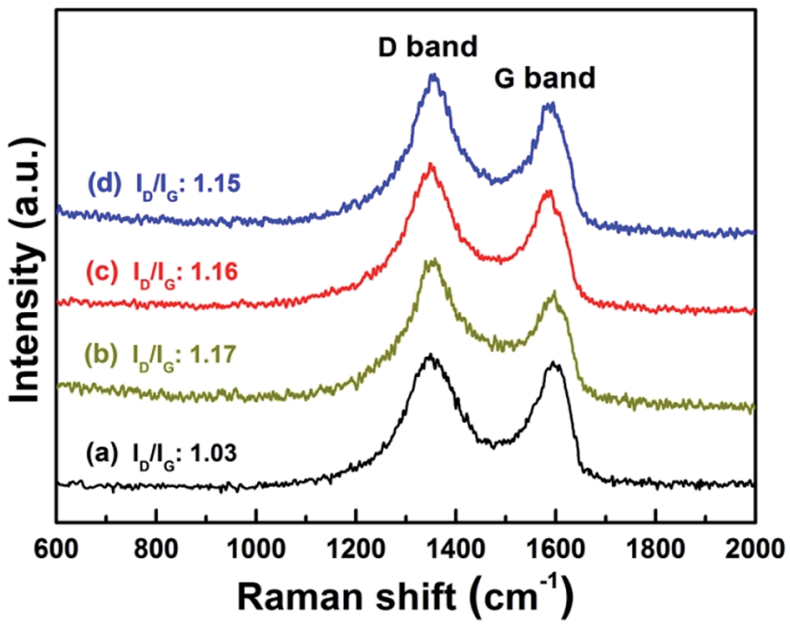

Fig. 2 Raman spectra of GO (a) and the Co-S/NS-rGO catalysts: (b) Co-S/NS-rGO-400, (c) Co-S/NS-rGO-500, (d) Co-S/NS-rGO-600.


Fig. 3 TEM and HR-TEM images of the catalysts synthesized at various temperatures: (a), (b) Co-S/NS-rGO-400; (c), (d) Co-S/NS-rGO-500; (e), (f) Co-S/NS-rGO-600.

typical TEM images of the composites synthesized at various temperatures. Clearly, the cobalt sulfide nanoparticles are irregular in shape and uniformly distributed on the GO surface for the Co-S/NS-rGO-400 sample. Average size of the $\mathrm{CoS}_{2}$ nanoparticles is about $15-20 \mathrm{~nm}$. And the lattice fringes with an interplanar spacing of $0.28 \mathrm{~nm}$ is in agreement with (200) plane of cubic $\mathrm{CoS}_{2}$ phase, as shown in the HR-TEM image (Fig. 2b). As the annealing temperature rises to above $500{ }^{\circ} \mathrm{C}$, the size and shape of nanoparticles on rGO surface does not show obvious changes. The lattice fringes of $0.19 \mathrm{~nm}$ and $0.29 \mathrm{~nm}$ correspond to the (102) and (100) planes of the hexagonal $\mathrm{Co}_{1-x} \mathrm{~S}$ phase, respectively, in good agreement with the XRD result.

Doping of heteroatoms into graphene was firstly analyzed by energy dispersive X-ray spectroscopy (EDS) equipped on FESEM. The EDS spectrum (Fig. S1 $\dagger$ ) demonstrates the presence of $\mathrm{N}, \mathrm{S}, \mathrm{Co}, \mathrm{O}$ and $\mathrm{C}$ elements in the as-synthesized Co-S/NSrGO composites. As shown in the EDS elemental mapping of the Co-S/NS-rGO-500 (Fig. 4), N, S and Co elements disperse homogeneously on the rGO sheet, confirming the successful doping of $\mathrm{N}$ and $\mathrm{S}$ in graphene and the uniform distribution of the cobalt sulfide nanoparticles on rGO surface. XPS analysis was also carried out to further study surface chemical composition of the obtained Co-S/NS-rGO composites. Fig. 5a shows survey spectra for the samples synthesized at various temperatures. The peaks around 285, 399, 532 and $782 \mathrm{eV}$ correspond to $\mathrm{C} 1 \mathrm{~s}, \mathrm{~N} 1 \mathrm{~s}, \mathrm{O}$ 1s and Co 2p, respectively. And the characteristic peaks appeared at 164 and $228 \mathrm{eV}$ are attributed to the presence of sulfur. Table 1 summarizes surface elemental composition of 

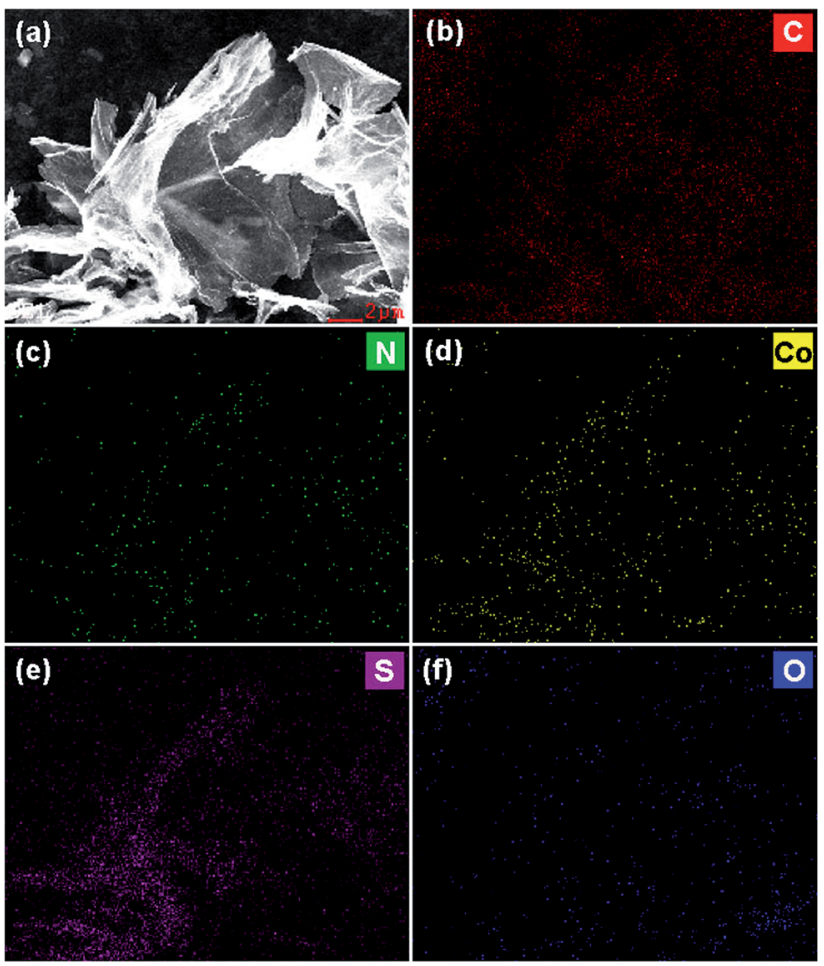

Fig. 4 SEM image (a) and elemental mapping analysis $(b-f)$ of the composite obtained at $500{ }^{\circ} \mathrm{C}$.
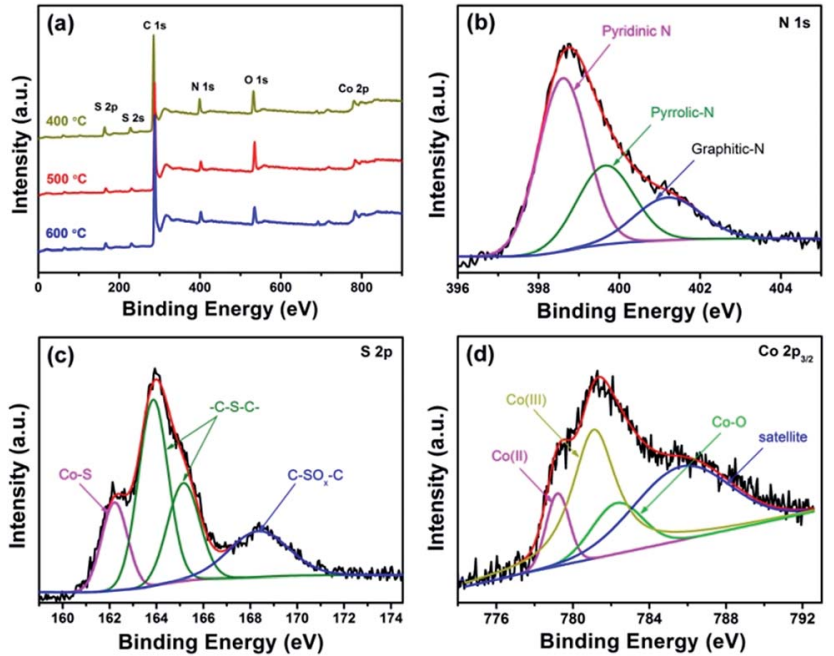

Fig. 5 (a) XPS survey spectra of the catalysts synthesized at various temperatures; high-resolution XPS spectra of the Co-S/NS-rGO-500 catalyst: (b) N 1s, (c) $S 2 p$, and (d) Co $2 p_{3 / 2}$.

the various samples. The $\mathrm{N}$ and $\mathrm{S}$ contents are determined to be 8.5 and 4.6 at\%, respectively, for the Co-S/NS-rGO-400 sample. With increasing the annealing temperature above $500{ }^{\circ} \mathrm{C}$, the composite shows significant decrease in the contents of $\mathrm{N}$ and $\mathrm{S}$.

High-resolution XPS spectra can provide more insight on the chemical state of each component. As shown in Fig. $5 \mathrm{~b}$, the $\mathrm{N} 1 \mathrm{~s}$ spectrum of the Co-S/NS-rGO-500 sample is deconvoluted into
Table 1 Surface elemental contents in various samples from XPS analysis

\begin{tabular}{llllll}
\hline Samples & Co (at\%) & S (at\%) & N (at\%) & C (at\%) & O (at\%) \\
\hline Co-S/NS-rGO-400 & 1.4 & 4.6 & 8.5 & 77.8 & 7.7 \\
Co-S/NS-rGO-500 & 1.1 & 2.5 & 5.7 & 81.4 & 9.3 \\
Co-S/NS-rGO-600 & 1.1 & 2.6 & 5.8 & 85.1 & 5.4
\end{tabular}

three peaks at $398.5,399.6$, and $401.1 \mathrm{eV}$, corresponding to pyridinic-N, pyrrolic-N, and graphitic-N, respectively. ${ }^{1,13,27,28}$ This provides further evidence that nitrogen has been doped in graphene matrix. Quantitative analysis from the $\mathrm{N} 1 \mathrm{~s}$ spectra of the various samples (Fig. S2 $\dagger$ ) reveals that, as the annealing temperature rises, relative contents of the three $\mathrm{N}$ species change. The largest pyridinic-N content is achieved for the CoS/NS-rGO-500 (55.3\%), while graphitic-N increases and pyrrolic$\mathrm{N}$ decreases with raising the temperature. In the high-resolution $\mathrm{S} 2 \mathrm{p}$ spectrum (Fig. 5c), the peak located at $162.5 \mathrm{eV}$ is attributed to the Co-S bond, while the pair of peaks at 163.8 and $164.9 \mathrm{eV}$ are assigned to $2 \mathrm{p}_{3 / 2}$ and $2 \mathrm{p}_{1 / 2}$ of thiophene-S (-C-S-C-) due to the spin-orbit coupling., ${ }^{2,20}$ The presence of thiophene-S reveals that the doped $\mathrm{S}$ atoms are directly bonded with two carbon atoms and defects of graphene. ${ }^{29}$ For all the composites obtained at various temperatures, the thiophene-S content is almost constant, over than 60 at\%, suggesting the stable incorporation of sulfur atoms in graphene framework. It was worth noting that an obvious peak at $168.4 \mathrm{eV}$ exists in the $\mathrm{S} 2 \mathrm{p}$ spectrum of the Co-S/NS-rGO, while the corresponding peak is absent for the NS-rGO (Fig. S3†), indicating covalent coupling between the cobalt sulfide nanoparticles and the NS-rGO through the $-\mathrm{C}-\mathrm{SO}_{x}-\mathrm{Co}-$ bonding. ${ }^{30}$ The relative contents of $\mathrm{N}$ and $\mathrm{S}$ components are presented in Table 2 for the composites annealed at various temperatures. Moreover, Co $2 \mathrm{p}_{3 / 2}$ peak at 779.1 and $781.2 \mathrm{eV}$ are assigned to $\mathrm{Co}^{\mathrm{III}}$ and $\mathrm{Co}^{\mathrm{II}}$, suggesting presence of the mixed oxidation state in the $\mathrm{Co}_{1-x} \mathrm{~S}$ phase, consistent with the previous reports. ${ }^{31,32}$ And the $\mathrm{Co}-\mathrm{O}$ peak appeared at $782.3 \mathrm{eV}$ can be ascribed to surface adsorption of $\mathrm{O}_{2}$ or slight oxidation of the cobalt sulfide nanoparticles exposed to air, further demonstrating the strong adsorption ability of the cobalt sulfide nanoparticles for oxygen molecules, which is beneficial for good ORR catalyst. ${ }^{33,34}$

The ORR activities of the Co-S/NS-RGO samples were firstly investigated by cyclic voltammetry in $0.1 \mathrm{M} \mathrm{KOH}$ solution at a potential scanning rate of $5 \mathrm{mV} \mathrm{s}^{-1}$. Compared with the featureless CVs obtained in $\mathrm{N}_{2}$-saturated solution, the CVs measured in $\mathrm{O}_{2}$-saturated electrolyte show pronounced oxygen reduction peaks for all the samples, indicating the obvious catalytic activity towards ORR (Fig. S3†). As shown in Fig. 6a, the ORR peak potential shifts from 0.786 to 0.836 and $0.799 \mathrm{~V}$ for the catalysts annealed at 400,500 and $600{ }^{\circ} \mathrm{C}$, respectively. The sample annealed at $500{ }^{\circ} \mathrm{C}$ exhibits the most positive peak potential, indicating the best ORR catalytic activity. Moreover, the ORR peak potential of $0.836 \mathrm{~V}$ is also more positive than

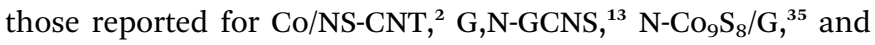
$\mathrm{CoS} / \mathrm{NSGA}^{36}{ }^{36}$ suggesting a more facile ORR process on this composite catalyst. 
Table 2 Relative contents of different components in N 1s and S 2p spectra for the various composites

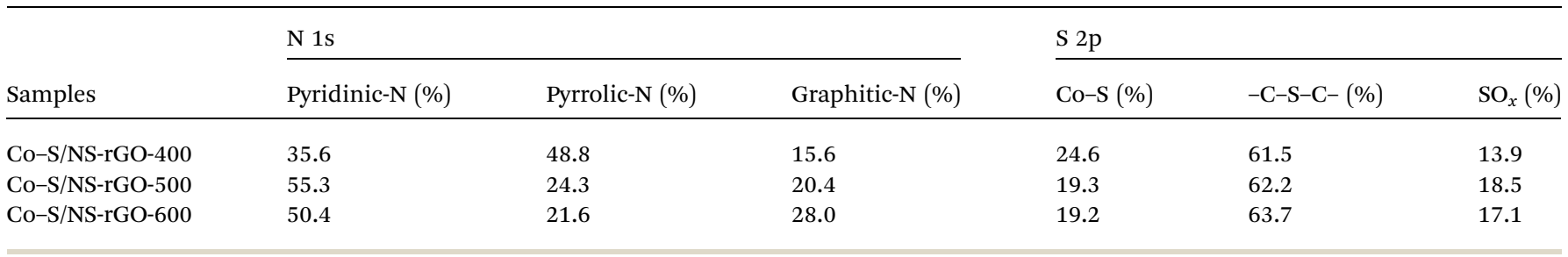
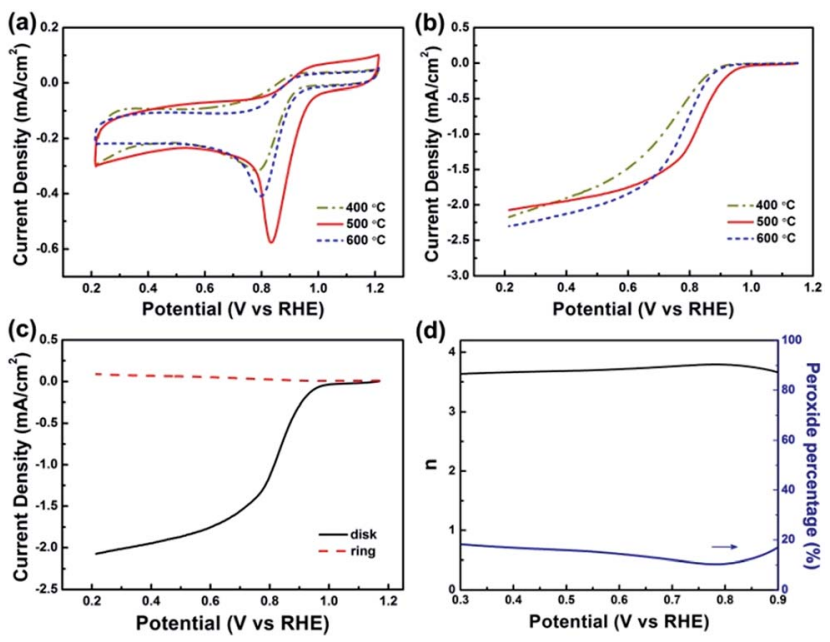

Fig. 6 Typical CVs (a) and LSV curves (b) of the Co-S/NS-rGO catalysts obtained at various temperatures in $\mathrm{O}_{2}$-saturated $0.1 \mathrm{M} \mathrm{KOH}$ solution; (c) ring and disk current on Co-S/NS-rGO-500 catalyst at rotation rate of $900 \mathrm{rpm}$ in $\mathrm{O}_{2}$-saturated $0.1 \mathrm{M} \mathrm{KOH}$ solution; (d) percentage of peroxide and the electron transfer number $(n)$ of the Co-S/NS-rGO-500 catalyst based on the corresponding RRDE data in (c).

Rotating disk electrode (RDE) measurements were carried out to further examine the impact of annealing temperature on ORR performance of the Co-S/NS-rGO catalysts at a scan rate of $5 \mathrm{mV} \mathrm{s}^{-1}$ with a rotating rate of $900 \mathrm{rpm}$. Fig. $6 \mathrm{~b}$ presents linear sweep voltammetry (LSV) curves of the Co-S/NS-rGO catalysts in $\mathrm{O}_{2}$-saturated 0.1 M KOH solution. Obviously, as the annealing temperature rises, the onset potential for ORR increases firstly from $0.903 \mathrm{~V}\left(400{ }^{\circ} \mathrm{C}\right)$ to $0.979 \mathrm{~V}\left(500{ }^{\circ} \mathrm{C}\right)$, and then decreases to $0.917 \mathrm{~V}\left(600{ }^{\circ} \mathrm{C}\right)$, whereas the half-wave potential shifts positively from $0.696 \mathrm{~V}\left(400{ }^{\circ} \mathrm{C}\right)$ to $0.811 \mathrm{~V}\left(500{ }^{\circ} \mathrm{C}\right)$, then shifts negatively to $0.756 \mathrm{~V}\left(600{ }^{\circ} \mathrm{C}\right)$. Therefore, from both the onset and half-wave potentials, the Co-S/NS-rGO-500 is determined to possess the highest catalytic activity towards ORR in $\mathrm{KOH}$ solution.

To gain further insight into the ORR process of the Co-S/NSrGO catalyst, rotating ring-disk electrode (RRDE) measurements were conducted on the Co-S/NS-rGO-500 catalyst. Fig. 6c shows ring current and disk current of the catalyst in $\mathrm{O}_{2}$-saturated $0.1 \mathrm{M} \mathrm{KOH}$ solution at a potential scan rate of $5 \mathrm{mV} \mathrm{s}^{-1}$ and a rotating rate of $900 \mathrm{rpm}$. The ring current is far less than the disk current, indicating that $\mathrm{H}_{2} \mathrm{O}$ is the main product of ORR process of this sample. The number of electrons transferred in per oxygen molecular $(n)$ and the percentage of peroxide yield $\left(\% \mathrm{HO}_{2}{ }^{-}\right)$during $\mathrm{ORR}$ can be calculated by the following equation: $:^{2,13}$

$$
\begin{gathered}
N=\frac{4 \times I_{\mathrm{d}}}{\left(I_{\mathrm{d}}+I_{\mathrm{r}} / N\right)} \\
\% \mathrm{HO}_{2}^{-}=200 \times \frac{I_{\mathrm{r}}}{N\left(I_{\mathrm{d}}+I_{\mathrm{r}} / N\right)}
\end{gathered}
$$

where $I_{\mathrm{d}}$ is the disk current, $I_{\mathrm{r}}$ is the ring current, and $N$ is the current collection efficiency of the Pt ring (0.37).

The calculated electron transfer number and the $\mathrm{HO}_{2}{ }^{-}$yield from the experimental results are presented in Fig. 6d. Clearly, the $n$ ranges between 3.61 and 3.79 in the potential range of $0.3-$ $0.9 \mathrm{~V}$, and the corresponding $\% \mathrm{HO}_{2}{ }^{-}$yield is less than $18 \%$, suggesting a four-electron dominated ORR process for this catalyst.

The superior ORR catalytic performance of the Co-S/NS-rGO500 composite catalyst is associated with the bonding configuration of the heteroatoms in rGO. As revealed by the aforementioned XPS analysis, the pyridinic-N content reaches the maximum $(55.3 \%)$ for the sample synthesized at $500{ }^{\circ} \mathrm{C}$, indicating close correlation between the ORR activity and the pyridinic-N species. ${ }^{37}$ Meanwhile, the thiophene-S incorporated in graphene also plays important role in the ORR performance. Although the exact active sites are still controversial, it is established that $\mathrm{N}$ doping into carbon allows charge delocalization and generates a net positive charge on the adjacent carbon atoms due to the large electronegativity of $\mathrm{N}$. The positively-charged carbon atoms may act as active sites for ORR to assist the adsorption of oxygen molecules. ${ }^{37,38}$ Moreover, the recent theoretical study reveals that $S$ doping may introduce spin density or increase charge density of carbon atoms at graphene edges and thus promote the ORR activity. ${ }^{39-41}$ Therefore, when $\mathrm{N}$ and $\mathrm{S}$ are dual doped into graphene, the synergistic effect may induce larger spin and charge density than single doping, exhibiting higher ORR catalyst activity.

To clarify the role of the cobalt sulfide nanoparticles in ORR process, the Co-S/NS-rGO-500 composite catalyst was compared with the nitrogen and sulfur dual doped rGO (NS-rGO) fabricated using the same procedure at $500{ }^{\circ} \mathrm{C}$. From the LSV curves in Fig. 7a, the NS-rGO shows an onset potential of $0.764 \mathrm{~V}$, much negative than that of the Co-S/NS-rGO-500. And the electron transfer number of the NS-rGO sample, 2.13-2.93 over the potential range from 0.3 to $0.9 \mathrm{~V}$, is also much lower than those of the Co-S/NS-rGO-500 catalyst (Fig. 7b). Therefore, it can be concluded that the $\mathrm{Co}_{1-x} \mathrm{~S}$ nanoparticles on the NS-rGO play important role in the ORR performance of the catalyst, which 



Fig. 7 LSV curves for oxygen reduction (a) and the electron transfer number (b) of the Co-S/NS-rGO-500 and NS-rGO in $\mathrm{O}_{2}$-saturated $0.1 \mathrm{M} \mathrm{KOH}$ solution.

not only provide more active sites to enhance the catalytic activity, ${ }^{19}$ but also facilitate the direct four-electron oxygen reduction process. Furthermore, covalent coupling between the $\mathrm{Co}_{1-x} \mathrm{~S}$ nanoparticles and the NS-rGO is believed to be important for excellent ORR activity of the Co-S/NS-rGO-500. Since the $\mathrm{Co}_{1-x} \mathrm{~S}$ nanoparticles are directly grown on $\mathrm{rGO}$, good electrical contact facilitates charge transfer between oxygen molecules absorbed on $\mathrm{Co}_{1-x} \mathrm{~S}$ active sites and the electrode. Besides, oxygen functional groups on rGO and defects induced by the heteroatom doping may promote heterogeneous nucleation of $\mathrm{Co}_{1-x} \mathrm{~S}$ on rGO and lead to smaller particle size with higher surface area, which further contributes to enhanced ORR performance. ${ }^{19}$ It is worth noting that the onset potential of $0.979 \mathrm{~V}$ for the Co-S/NS-rGO-500 sample, although still inferior to that of the $\mathrm{Pt} / \mathrm{C}$ catalyst, is higher than previously reported cobalt chalcogenide electrocatalysts in $\mathrm{KOH}$ electrolyte, such as $\mathrm{Co}_{3} \mathrm{~S}_{4} / \mathrm{G}(0.92 \mathrm{~V}){ }_{1}^{18} \mathrm{Co}_{1-x} \mathrm{~S} / \mathrm{rGO}(0.87 \mathrm{~V}),{ }^{19} \mathrm{Co}_{9} \mathrm{~S}_{8} / \mathrm{NHCS}(0.97 \mathrm{~V}){ }^{33}$ $\mathrm{CoS}_{2} / \mathrm{N}, \mathrm{S}-\mathrm{GO}(0.97 \mathrm{~V}),{ }^{32}$ and $\mathrm{N}-\mathrm{Co}_{9} \mathrm{~S}_{8} / \mathrm{G}(0.941 \mathrm{~V}),{ }^{35}$ highlighting the excellent ORR catalytic activity of our composite catalyst.

Loading amount of the $\mathrm{Co}_{1-x} \mathrm{~S}$ nanoparticles on graphene was optimized to further improve the ORR performance. Three Co-S/NS-rGO-500 samples were fabricated by using different amount of $\mathrm{Co}(\mathrm{OAC})_{2}$ as the precursor at $500{ }^{\circ} \mathrm{C}$. As shown in Fig. 8 , the ORR peak potential first shifts positively from 0.822 to $0.836 \mathrm{~V}$, then shifts negatively to $0.722 \mathrm{~V}$ with increasing the $\mathrm{Co}(\mathrm{OAC})_{2}$ mass. Therefore, the Co-S/NS-rGO composite catalyst synthesized with $5.0 \mathrm{mg}$ cobalt precursor manifests the highest catalytic activity towards ORR.

To evaluate long-term stability of the Co-S/NS-rGO-500 catalyst, current-time $(i-t)$ chronoamperometry measurement was carried out at a constant potential of $0.68 \mathrm{~V}$ in the $\mathrm{O}_{2}$ saturated $0.1 \mathrm{M} \mathrm{KOH}$ solution with a rotating speed of 1600 rpm. As shown in Fig. 9, even after 21000 s, the Co-S/NSrGO-500 catalyst exhibits a very slow attenuation with a high current retention (about 94\%), which also outperforms the stability of $\mathrm{Co}_{x} \mathrm{~S}_{y} @ \mathrm{C}-1000,{ }^{1} \mathrm{Co}_{9} \mathrm{~S}_{8} / \mathrm{NHCS}-900,{ }^{32} \mathrm{CoS} / \mathrm{NSGA},{ }^{36}$ and $\mathrm{Pt} / \mathrm{C}$ catalyst ${ }^{1}$ reported previously.

Since ORR in acid media is more challenging for nonprecious metal catalysts, ORR performance of the Co-S/NSrGO composites synthesized at various temperatures was also investigated by $\mathrm{RDE}$ and $\mathrm{RRDE}$ measurements in $\mathrm{H}_{2} \mathrm{SO}_{4}$ medium. Fig. 10a shows LSV curves of the Co-S/NS-rGO catalysts in $\mathrm{O}_{2}$-saturated $0.5 \mathrm{M} \mathrm{H}_{2} \mathrm{SO}_{4}$ solution. Clearly, with the increase of the annealing temperature, the onset potential

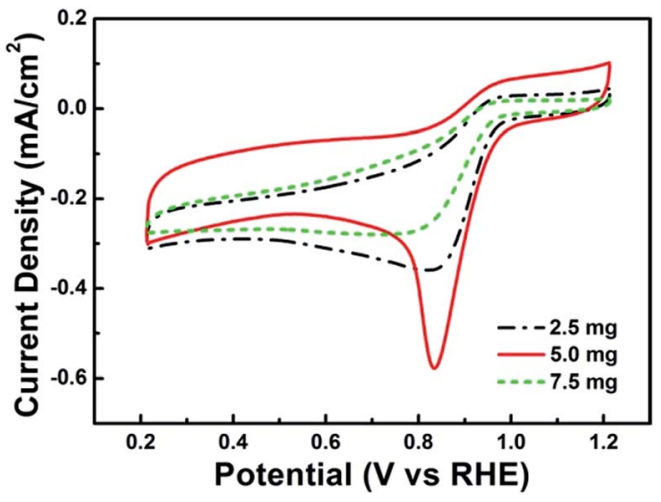

Fig. 8 Electrocatalytic stability of the Co-S/NS-rGO-500 catalysts in $\mathrm{O}_{2}$-saturated $0.1 \mathrm{M} \mathrm{KOH}$ solution at $0.68 \mathrm{~V}$ (vs. $\mathrm{RHE}$ ) with a rotation rate of $1600 \mathrm{rpm}$.



Fig. 9 CV curves of the Co-S/NS-rGO-500 catalysts synthesized with different amount of $\mathrm{Co}(\mathrm{OAC})_{2}$ in $\mathrm{O}_{2}$-saturated $0.1 \mathrm{M} \mathrm{KOH}$ solution.
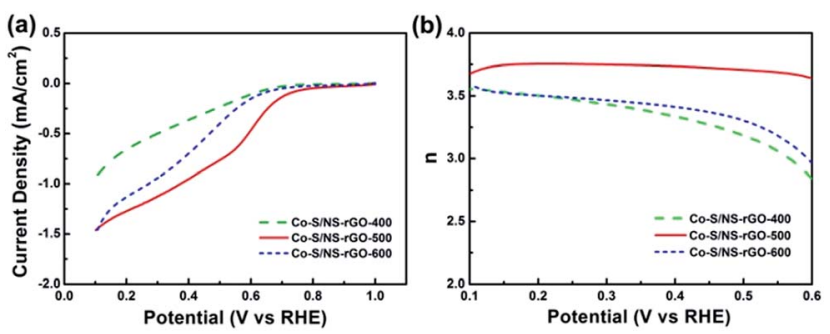

Fig. 10 LSV curves (a) and the electron transfer number (b) of various Co-S/NS-rGO catalysts in $\mathrm{O}_{2}$-saturated $0.5 \mathrm{M} \mathrm{H}_{2} \mathrm{SO}_{4}$ solution at a scan rate of $5 \mathrm{mV} \mathrm{s}^{-1}$ and a rotation rate of $900 \mathrm{rpm}$.

firstly increases from $0.663 \mathrm{~V}\left(400{ }^{\circ} \mathrm{C}\right)$ to $0.791 \mathrm{~V}\left(500{ }^{\circ} \mathrm{C}\right)$, then shift negatively to $0.698 \mathrm{~V}\left(600{ }^{\circ} \mathrm{C}\right)$. A similar tendency is also found in the half-wave potential. Accordingly, the Co-S/NS-rGO500 sample is proved to show the best catalytic activity in acid solution. Moreover, from RRDE tests, the electron transfer number of 3.64-3.75 is determined in the potential range of 0.1-0.6 V for the catalyst obtained at $500{ }^{\circ} \mathrm{C}$ (Fig. 10b), suggesting the good selectivity for four-electron reduction pathway in the acid solution. Although the catalytic activity and selectivity of the Co-S/NS-rGO-500 are still inferior to those of the Pt/ 
C catalyst in acid media, ${ }^{\mathbf{4 2}}$ the ORR activity with an onset potential of $0.791 \mathrm{~V}$ is among the best results for cobalt chalcogenide catalysts in acid electrolyte. ${ }^{\mathbf{1 9 , 4 2}}$

Overall, the prominent ORR performance of the Co-S/NSrGO-500 catalyst in both alkaline and acid electrolyte is attributed to the following advantages. Firstly, dual-doping of $\mathrm{N}$ and $\mathrm{S}$ creates more active sites in graphene for ORR. Secondly, the $\mathrm{Co}_{1-x} \mathrm{~S}$ nanoparticles with small size have higher surface area and expose more active sites. Thirdly, covalent coupling between the NS-rGO sheet and the $\mathrm{Co}_{1-x} \mathrm{~S}$ nanoparticles facilitates charge transfer between the absorbed oxygen molecules and the electrode. Finally, close interaction and covalent bonding of the rGO with the $\mathrm{Co}_{1-x} \mathrm{~S}$ nanoparticles prevents the detachment of the catalyst particles from the conducting graphene surface, which favors the long-term stability.

\section{Conclusions}

A high-efficiency Co-S/NS-rGO composite electrocatalyst was developed successfully from graphene oxide, $\mathrm{NH}_{4} \mathrm{SCN}$ and $\mathrm{Co}(\mathrm{OAC})_{2}$ precursors by a facile one-step annealing process. Depending on the temperature, $\mathrm{CoS}_{2}$ or $\mathrm{Co}_{1-x} \mathrm{~S}$ nanoparticles were grown on graphene support, and nitrogen and sulfur were simultaneously incorporated into rGO. Due to the synergistic effect of the cobalt sulfide nanoparticles and the $\mathrm{N}, \mathrm{S}$ dualdoped graphene, the Co-S/NS-rGO-500 catalyst exhibits excellent ORR activity in $\mathrm{O}_{2}$-saturated $0.1 \mathrm{M} \mathrm{KOH}$ solution with a peak potential of $0.836 \mathrm{~V}$ and an onset potential of $0.979 \mathrm{~V}$, comparable to that of commercial $\mathrm{Pt} / \mathrm{C}$ catalyst. And this catalyst manifests good four-electron selectivity and robust longterm stability. Besides, in $0.5 \mathrm{M} \mathrm{H}_{2} \mathrm{SO}_{4}$ medium, the Co-S/NSrGO-500 also shows the best ORR performance among all samples. The facile synthesis method and the superior ORR performance of the Co-S/NS-rGO composites demonstrate great promise for application in fuel cells and metal-air batteries.

\section{Conflicts of interest}

There are no conflicts to declare.

\section{Acknowledgements}

This work was financially supported by the Natural Science Foundation of Shanghai (16ZR1423500) and The Program for Associate Professor of Special Appointment (Young Eastern Scholar) at Shanghai Institutions of Higher Learning (QD2016013).

\section{Notes and references}

1 B. Chen, R. Li, G. Ma, X. Gou, Y. Zhu and Y. Xia, Nanoscale, 2015, 7, 20674-20684.

2 Y. Liu, B. Zhao, Y. Zhang, H. Zhang, K. Zhan, J. Yang and J. Li, RSC Adv., 2016, 6, 32676-32684.

3 M. Shen, C. Ruan, Y. Chen, C. Jiang, K. Ai and L. Lu, ACS Appl. Mater. Interfaces, 2015, 7, 1207-1218.
4 W. Choi, G. Yang, S. L. Kim, P. Liu, H. J. Sue and C. Yu, J. Power Sources, 2016, 313, 128-133.

5 F. Jaouen, E. Proietti, M. Lefevre, R. Chenitz, J.-P. Dodelet, G. Wu, H. T. Chung, C. M. Johnston and P. Zelenay, Energy Environ. Sci., 2011, 4, 114-130.

6 L. Zhang and Z. Xia, J. Phys. Chem. C, 2011, 115, 1117011176.

7 S. Chen, J. Bi, Y. Zhao, L. Yang, C. Zhang, Y. Ma, Q. Wu, X. Wang and Z. Hu, Adv. Mater., 2012, 24, 5593-5597.

8 K. Gong, F. Du, Z. Xia, M. Durstock and L. Dai, Science, 2009, 323, 760-764.

9 J. P. Paraknowitsch, Energy Environ. Sci., 2013, 6, 2839-2855.

10 L. Qu, Y. Liu, J. B. Baek and L. Dai, ACS Nano, 2010, 4, 13211326.

11 L. Lai, J. R. Potts, D. Zhan, L. Wang, C. K. Poh, C. Tang, H. Gong, Z. Shen, J. Lin and R. S. Ruoff, Energy Environ. Sci., 2012, 5, 7936-7942.

12 J. Liang, Y. Jiao, M. Jaroniec and S. Z. Qiao, Angew. Chem., Int. Ed., 2012, 51, 11496-11500.

13 R. Li, Z. Wei and X. Gou, ACS Catal., 2015, 5, 4133-4142.

14 Y. Feng, T. He and N. Alonsovante, Chem. Mater., 2008, 20, 26-28.

15 Y. X. Zhou, H. B. Yao, Y. Wang, H. L. Liu, M. R. Gao, P. K. Shen and S. H. Yu, Chem.-Eur. J., 2010, 16, 1200012007.

16 M. R. Gao, Y. F. Xu, J. Jiang and S. H. Yu, Chem. Soc. Rev., 2013, 44, 2986-3017.

17 Q. Liu and J. Zhang, CrystEngComm, 2013, 15, 5087-5092.

18 N. Mahmood, C. Zhang, J. Jiang, F. Liu and Y. Hou, Chem.Eur. J., 2013, 19, 5183-5190.

19 H. Wang, Y. Liang, Y. Li and H. Dai, Angew. Chem., Int. Ed., 2011, 50, 10969-10972.

20 D. Higgins, F. M. Hassan, H. S. Min, J. Y. Choi, M. A. Hoque, U. L. Dong and Z. Chen, J. Mater. Chem. A, 2015, 3, 63406350.

21 Z. Luo, B. Zhao, Y. Liu, H. Zhang, Z. Tang, J. Li and J. Yang, Electrochim. Acta, 2015, 161, 72-79.

22 L. Lin, Q. Zhu and A. W. Xu, J. Am. Chem. Soc., 2014, 136, 11027-11033.

23 Y. X. Wang, S. L. Chou, H. K. Liu and S. X. Dou, Carbon, 2013, 57, 202-208.

24 B. Zhao, L. Zhang, X. Wang and J. Yang, Carbon, 2012, 50, 2710-2716.

25 Y. Zheng, Y. Jiao, L. Ge, M. Jaroniec and S. Z. Qiao, Angew. Chem., Int. Ed., 2013, 125, 3192-3198.

26 X. Gu, C. J. Tong, C. Lai, J. Qiu, X. Huang, W. Yang, B. Wen, L. M. Liu, Y. Hou and S. Zhang, J. Mater. Chem. A, 2015, 3, 16670-16678.

27 J. Sanetuntikul, C. Chuaicham, Y. Choi and S. Shanmugam, J. Mater. Chem. A, 2015, 3, 15473-15481.

28 J. Sanetuntikul and S. Shanmugam, Nanoscale, 2015, 7, 7644-7650.

29 S. Gao, L. Li, K. Geng, X. Wei and S. Zhang, Nano Energy, 2015, 16, 408-418.

30 W. Yan, X. Cao, J. Tian, C. Jin, K. Ke and R. Yang, Carbon, 2016, 99, 195-202. 
31 J. Zhu, Z. Ren, S. Du, Y. Xie, J. Wu, H. Meng, Y. Xue and H. Fu, Nano Res., 2017, 10, 1819-1831.

32 P. Ganesan, M. Prabu, J. Sanetuntikul and S. Shanmugam, ACS Catal., 2015, 5, 3625-3637.

33 J. Wang, L. Li, X. Chen, Y. Lu and W. Yang, J. Mater. Chem. A, 2016, 4, 11342-11350.

34 Q. Wang, L. Jiao, H. Du, Y. Si, Y. Wang and H. Yuan, J. Mater. Chem., 2012, 22, 21387-21391.

35 S. Dou, L. Tao, J. Huo, S. Wang and L. Dai, Energy Environ. Sci., 2016, 9, 1320-1326.

36 Z. Luo, C. Tan, X. Zhang, J. Chen, X. Cao, B. Li, Y. Zong, L. Huang, X. Huang and L. Wang, Small, 2016, 12, 59205926.
37 S. Yang, L. Zhi, K. Tang, X. Feng, J. Maier and K. Mullen, Adv. Funct. Mater., 2012, 22, 3634-3640.

38 S. Bag, B. Mondal, A. K. Das and C. R. Raj, Electrochim. Acta, 2015, 163, 16-23.

39 J. Xu, G. Dong, C. Jin, M. Huang and L. Guan, ChemSusChem, 2013, 6, 493-499.

40 Z. Cui, S. Wang, Y. Zhang and M. Cao, J. Power Sources, 2014, 259, 138-144.

41 I. Y. Jeon, S. Zhang, L. Zhang, H. J. Choi, J. M. Seo, Z. Xia, L. Dai and J. B. Baek, Adv. Mater., 2013, 25, 6138-6145.

42 W. Gu, L. Hu, W. Hong, X. Jia, J. Li and E. Wang, Chem. Sci., 2016, 7, 4167-4173. 